\title{
Biobran/MGN-3, an arabinoxylan rice bran, enhances NK cell activity in geriatric subjects: A randomized, double-blind, placebo-controlled clinical trial
}

\author{
AHMED F. ELSAID ${ }^{1}$, MAGDA SHAHEEN ${ }^{2}$ and MAMDOOH GHONEUM ${ }^{3}$ \\ ${ }^{1}$ Department of Community Medicine and Public Health, Faculty of Medicine, \\ Zagazig University, Zagazig, Al Sharqia 44519, Egypt; Departments of ${ }^{2}$ Internal Medicine and \\ ${ }^{3}$ Surgery, Charles R. Drew University of Medicine and Science, Los Angeles, CA 90059, USA
}

Received June 11, 2016; Accepted November 28, 2017

DOI: $10.3892 /$ etm.2018.5713

\begin{abstract}
Aging is associated with a decline in natural killer (NK) and natural killer T (NKT) cell function that may contribute to increased susceptibility to malignancy and infection. A preliminary investigation was conducted examining the hypothesis that arabinoxylan rice bran (Biobran/MGN-3), a denatured hemicellulose with known immunomodulatory activity, could counteract this decline in NK/NKT cell activity in geriatrics. A total of 12 healthy geriatric subjects of both sexes and over 56 years old, participated in a randomized, double-blind, placebo-controlled clinical trial. A total of six subjects served as control and six subjects ingested Biobran/MGN-3 (500 mg/day) for 30 days. The effect of Biobran/MGN-3 supplementation on NK/NKT cell activity was assessed using the degranulation assay. All study subjects were monitored for the development of any inadvertent side effects. In addition, the pharmacological effects of Biobran/MGN-3 on blood cell components and liver and kidney functions were also assessed. Results demonstrated that Biobran/MGN-3 had no effect on the total percentage of NK cells, however it enhanced the cytotoxic activity of induced NK cell expression of cluster of differentiation 107a, when compared with baseline values and with the placebo group $(\mathrm{P}<0.05)$. Furthermore, there were no side effects observed, indicating that Biobran/MGN-3 supplementation was safe at the utilized dosage and for the duration of administration. Various additional beneficial effects were observed, including improved mean corpuscular volume and reduced hepatic aspartate aminotransferase enzyme levels, which suggested improved liver function. It was concluded that Biobran/MGN-3 induces
\end{abstract}

Correspondence to: Dr Ahmed F. Elsaid, Department of Community Medicine and Public Health, Faculty of Medicine, Zagazig University, El-Gamaah Street, Zagazig, Al Sharqia 44519, Egypt

E-mail: elsaid@ucla.edu; elsaid_af@hotmail.com

Key words: Biobran/MGN-3, geriatric, NK cells, NKT cells, degranulation a significant increase in NK activity which may increase resistance to viral infections and cancers in the geriatric population. However, additional clinical trials should be conducted in the future to verify these findings.

\section{Introduction}

The geriatric population is rapidly increasing worldwide. According to the World Health Organization, the number of people aged 65 and older is expected to increase to 1.5 billion by 2050 and to significantly outnumber the population of children 5 years old and younger (1). In 2012, the per person health care spending of individuals 65 and older was approximately 5 times higher than for children and 3 times higher than for working-aged persons. Significant resources are devoted to maintaining the health of this geriatric population, which is prone to increased incidences of infections, cancers, and degenerative diseases. For example, the estimated healthcare costs for individuals with Alzheimer's and other dementias in 2017 is 259 billion USD (2), and the cost for those with cancer is 87.8 billion USD (3).

Immunodeficiency in the geriatric population plays a major role in their increased susceptibility to infections, cancers, and neurodegeneration. Recent research has found evidence suggesting that the nervous system and immune system are intimately linked and engage in significant cross-talk to preserve homeostasis. Several proteins that were originally thought to be exclusive to the immune system have been detected in the healthy nervous system (4), and the term synaptoimmunology has been used recently to characterize the interplay between immune modulators and synaptic function (5). The link between decreased immunological vigor and increased infection and cancer has been substantiated in many studies which show the reduction of leukocyte qualitative functions during aging (6-9). Of particular interest to the study of immunodeficiency in the geriatric population is the reported age-dependent reduction of natural killer (NK) cells which constitute the primary line of defense against malignant and virally-infected cells (10-13). Enhancing NK cell activity is therefore an essential therapeutic target in the geriatric population. 
Given the significant costs mentioned above, attempts need to be made to find natural, safe, and inexpensive agents to improve age-associated health and treatment of diseases. In the current preliminary study, we hypothesized that the natural dietary supplement arabinoxylan derived from rice bran, Biobran/MGN-3, could counteract the age-induced decline of NK cell activity in geriatric subjects over 56 years old. Biobran/MGN-3 is manufactured by hydrolyzing rice bran with the enzymatic extract of Shiitake mushrooms (14) and several studies have demonstrated its potent immunomodulatory effect (15-20). For example, Biobran/MGN-3 has been shown to enhance NK cell activity by 2-3 fold in young adults (human subjects aged 20-46 years) (21). The effect was dose and time dependent and was associated with an increase in the binding capacity of NK cells to tumor cell targets (21). In addition, the positive effects of Biobran/MGN-3 on human T and B cell mitogen response have also been examined in vivo (14).

To test our hypothesis, we conducted a one-month, randomized, double-blind, placebo-controlled clinical trial in geriatric subjects attending Zagazig University Hospital in Zagazig, Al Sharqia, Egypt. The aim of our study was to evaluate the response of these geriatric subjects to the immune modulatory effect of Biobran/MGN-3 and to determine whether the previously reported positive effects of Biobran/MGN-3 supplementation on NK cell activity could be extended to this population.

\section{Subjects and methods}

Biobran/MGN-3 is a denatured hemicellulose obtained by reacting rice bran hemicellulose with multiple carbohydrate hydrolyzing enzymes from Shiitake mushrooms. It is an arabinoxylane with a xylose in its main chain and an arabinose polymer in its side chain (14). $500 \mathrm{mg}$ of Biobran/MGN-3 was ingested once per day in the form of powder enclosed in sachets. The control group ingested the same amount of placebo powder, which was indistinguishable from Biobran/MGN-3 in terms of color, smell, and consistency. The sachets of Biobran/MGN-3 and placebo were indistinguishable except for a letter code imprinted on the bottom of the sachets. Both Biobran/MGN-3 and placebo sachets were kindly provided by Daiwa Pharmaceuticals Co., Ltd., Tokyo, Japan.

Ingredients of the study materials. Biobran/MGN-3 group sachets contained Biobran/MGN-3 (500 mg), Maltitol (1,000 mg), Dextrin (200 mg), Hydroxypropyl Distarch Phosphate (280 mg), and Tricalcium Phosphate (20 mg). Placebo group sachets contained Maltitol (1,000 mg), Dextrin (200 mg), Hydroxypropyl Distarch Phosphate (780 mg), and Tricalcium Phosphate $(20 \mathrm{mg})$.

\section{Inclusion/exclusion criteria for patients}

Inclusion criteria. Included in the study were subjects 56 years or older who were willing to provide written consent to participate voluntarily in the study.

Exclusion criteria. We excluded subjects with current or a history of infections or malignancies, auto-immune disorders, marked portal hypertension and pancytopenia, or major psychological insult. We also excluded those who were using vitamin or antibiotic supplements, women who were lactating, and patients receiving other anti-viral or anticancer therapies (radiation, chemotherapy).

Study subjects and design. Twelve apparently healthy geriatric subjects ( $\geq 56$ years old) of both sexes ( 6 males and 6 females) were randomly selected from visitors of outpatient clinics at Zagazig University Hospital, Zagazig, Egypt. Subjects were randomly assigned to either the Biobran/MGN-3 group ( $\mathrm{n}=6,500 \mathrm{mg} / \mathrm{day}$ orally) or the placebo control group ( $\mathrm{n}=6,500 \mathrm{mg} / \mathrm{day}$ orally) using the random sample selection function of SPSS. Only the Principal Investigator (PI) had the code for Biobran/MGN-3 and placebo groups. Each participant gave 2 blood samples ( $4 \mathrm{ml}$ each) at the initiation and termination of the study for laboratory and flow cytometry investigation. During the study, enrolled subjects were asked not to take any over-the-counter drugs, including vitamins, without consulting with the study PI. All participants were asked to report any eruption of abnormal symptoms or signs to the PI. The study protocol conformed to the ethical guidelines of the 1975 Declaration of Helsinki and was approved by Institutional Review Board (IRB approval no. 1507, June 2015), Zagazig University Hospital, Faculty of Medicine.

Laboratory investigations. This included red blood cells (RBC), hematocrit (HCT), hemoglobin ( $\mathrm{Hb})$, mean corpuscular hemoglobin $(\mathrm{MCH})$, mean corpuscular volume (MCV), platelet count, white blood cells (WBC), neutrophils, and basophils + eosinophils. Laboratoy investigations of liver and kidney function included alanine aminotransferase (serum glutamic pyruvic transaminase) (ALT/SGPT), aspartate aminotransferase (serum glutamic oxaloacetic transaminase) (AST/SGOT), and uric acid (UA). ALT, AST, ALP, and UA levels were measured by photometric methods using COBAS Integra 400 plus Analyzer (Roche, Basel, Switzerland).

Degranulation assay. Blood was dispensed at a volume of $100 \mu \mathrm{l}$ into each of 4 tubes to serve as stimulated, unstimulated control, positive control, or IgG isotypic negative control. Stimulation of NK/NKT cells was conducted chemically by incubation $\left(5 \mathrm{~h}, 37^{\circ} \mathrm{C}\right.$, under $\left.5 \%(\mathrm{v} / \mathrm{v}) \mathrm{CO}_{2}\right)$ with a combination of Phorbol-12-myristate-13-acetate (PMA, $50 \mathrm{ng} / \mathrm{ml}$; Sigma-Aldrich; Merck KGaA, Darmstadt, Germany) and $\mathrm{Ca}^{2+}$ Ionophore (Ionomycin, $250 \mathrm{ng} / \mathrm{ml}$, Sigma) in a final volume of $500 \mu \mathrm{l}$ (adjusted with RPMI-1640 medium). Unstimulated samples were incubated with RPMI-1640 medium without PMA/Ionomycin stimulation, whereas in positive control samples, cytochalasin (Sigma-Aldrich; Merck KGaA) was added in conjunction with PMA/Ionomycin. FITC-labeled mouse anti-human CD-107a clone H4A3 (BD Bioscience, San Jose, CA, USA) was added during PMA/Ionomycin stimulation. Monensin (GolgiStop; BD Bioscience, San Jose, CA, USA) was added to a final concentration of $6 \mu \mathrm{g} / \mathrm{ml} 1 \mathrm{~h}$ after incubation to block internalization and degradation of CD107a. In the IgG isotypic negative control tube, mouse anti-human FITC-labeled IgG AB (clone G18-145; BD Bioscience) was added instead of CD107a (22).

After incubation, RBCs were lysed with BD PharmLyse (BD Bioscience) before cells were collected by spinning, washed once with staining buffer, and re-suspended in $100 \mu 1$ staining 
buffer. Cells were stained by incubation with $5 \mu 1$ of PE-labeled mouse anti-human CD56 AB clone R19-760 (NCAM-1, BD Bioscience) and $5 \mu \mathrm{l}$ of PerCP-labeled mouse anti-human CD3 clone SK7 (BD Bioscience) for $15 \mathrm{~min}$ at $37^{\circ} \mathrm{C}$. Cells were then collected by spinning, washed once with FACS buffer, and then re-suspended in FACS buffer. Analysis was carried out using BD FACSCalibur with CellQuest software (BD Bioscience).

Sample size. A power analysis using a large effect size of 0.80 for a repeated measure analysis of variance with two measurements in 2 groups was performed using $\mathrm{G}^{*}$ Power software package (Version 3.1.9.2; Franz Faul, Germany). With the criterion of significance $(\alpha)$ set at 0.05 , a sample size of 6 subjects per group (total of 12 subjects) results in a power of 0.82 to yield a statistically significant result.

Statistical analysis. Continuous variables were expressed as means \pm standard deviation. Two-tailed Student's t-test or Fisher exact test were used to compare between groups, whereas two-tailed paired t-test was used to examine the effect of treatment within a group (i.e., compare post-treatment level with the pre-treatment level). We used Shapiro-Wilks test to determine whether the data were normally distributed. For non-normally distributed data, we used non-parametric test, Wilcoxon Singed Rank test, and Mann-Whitney test to examine the effect of treatment within and between groups, respectively. $\mathrm{P}<0.05$ was considered to indicate a statistically significant difference. To produce graphs, the mean $\pm 95 \%$ confidence interval was used. SPSS version 22 (IBM Corp., Armonk, NY, USA) and GraphPad Prism 6 (GraphPad Software, Inc., La Jolla, CA, USA) were used for analysis.

\section{Results}

Hematological and biochemical profile. Different base-line blood parameters, liver enzymes, and kidney function were examined in 12 geriatric subjects of both Biobran/MGN-3 and placebo groups. Data in Tables I and II show that there was no statistically significant difference between the two groups $(\mathrm{P}>0.05)$ in all baseline variables except for AST level $(\mathrm{P}=0.041)$. Table I further shows that there was no significant difference between baseline values and values after 1 month of supplementation for the measured characteristics in the Biobran/MGN-3 group, except for levels of $\mathrm{Hb}, \mathrm{MCV}, \mathrm{MCH}$ and AST $(\mathrm{P}<0.05)$. Table II shows that there was no significant difference between baseline values and values after 1 month of supplementation for the measured characteristics in the Placebo group, except for levels of $\mathrm{Hb}$ and $\mathrm{MCH}(\mathrm{P}<0.05)$. This is further supported by lack of observation of any side effects in the Biobran/MGN-3 group, indicating that its treatment was safe at the used dosage and duration. Instead, some beneficial effects were observed after one month treatment in the Biobran/MGN-3 group, and the placebo group to a lesser extent. These included improved levels of $\mathrm{Hb}, \mathrm{MCV}$, and $\mathrm{MCH}$ from pre-treatment values. In addition, Biobran/MGN-3 supplementation significantly down-regulated AST (SGOT) levels, suggesting improved liver functions.

Percentage of lymphocyte, NK, and NKT cells. Data in Table III show that basal values (before treatment) of percentage of lymphocyte, NK, and NKT cells were not significantly different between the Biobran/MGN-3 and placebo groups ( $\mathrm{P}>0.05)$. Furthermore, Table III shows that Biobran/MGN-3 and placebo supplementation for 1 month did not induce significant changes in the percentage of lymphocyte, NK, and NKT cells when compared with their baseline values $(\mathrm{P}>0.05)$. The observed increase in the \% lymphocytes is within the normal range in adults and aged humans (18-44\%).

NK cells expressing CD107a. Flow cytometric analysis was carried out to examine the effect of Biobran/MGN-3 on CD107a degranulation in PMA/Ionomycin activated NK cells. Supplementation with Biobran/MGN-3 significantly upregulated stimulated PMA/Ionomycin-induced CD107a-expressing NK cells (Fig. 1 and Table IV). Both groups showed similar changes in the levels of CD107a expression in PMA/Ionomycin-stimulated NK cells before initiation of treatment. It is important to note that the comparison of the percentages of pre-treatment levels of PMA/Ionomycin-activated NK cells that express CD107a in Biobran/MGN-3 group (60.5\%) and placebo group (40.9\%) (Table IV) was not significant using Mann-Whitney U test (results of test not shown).

Furthermore,Fig.2showstheeffectofBiobran/MGN-3 supplementation on upregulating the percentage of stimulated CD107a expressing NK cells. The number of PMA/Ionomycin-stimulated NK cells that express CD107a was significantly higher than baseline values. On the other hand, supplementation for the placebo group did not upregulate PMA/Ionomycin-stimulated NK expressing CD107a. CD107a was positive only in very few NK cells in the placebo subjects (Fig. 2). The data suggest that Biobran/MGN-3 supplementation induces NK cell expression of CD107a, which has been reported to parallel an increase in the cytotoxic effect of NK cells.

NKT cells expressing CD107a. Biobran/MGN-3 and placebo supplementation did not significantly upregulate the percentages of PMA/Ionomycin-stimulated NKT (CD3+ve, CD56+ve) cells expressing CD107a as compared to their baseline values (Fig. 3 and Table IV).

\section{Discussion}

Biobran/MGN-3 is an arabinoxylan derived from rice bran. It has a xylose in its main chain and an arabinose polymer in its side chain (14). Biobran/MGN-3 is a potent biological response modifier (BRM) as manifested by its ability to increase NK cell activity and IFN- $\gamma$ production by peripheral blood lymphocytes in vitro (15) and to enhance NK activity in young adult humans (21) and cancer patients (19,23,24). Biobran/MGN-3 also enhances NK activity in animals, including aged mice (25) and animal bearing tumor $(17,26)$. This increase in activity was associated with an increase in both the granular content of NK cells and their binding capacity to tumor cell targets $(21,25,27)$. Further studies on NK function in geriatric subjects are needed to ascertain the benefits of this BRM on aged humans.

NK cells represent the first line of defense against cancer and virally infected cells, due to the fact that their cytotoxic activity directly kills target cells. However, the impairment of $\mathrm{NK}$ activity due to aging has been reported in both experimental animals and humans, and renders the geriatric population more susceptible to viral infection and malignancies $(9,28-32)$. 
Table I. Hematological and biochemical characteristics (blood parameters, liver enzymes, and kidney function) of geriatric subjects in the Biobran/MGN-3 group.

\begin{tabular}{|c|c|c|c|}
\hline \multirow[b]{2}{*}{ Parameters } & \multicolumn{2}{|c|}{ Biobran/MGN-3 Group } & \multirow[b]{2}{*}{ P-value } \\
\hline & Baseline & 1 Month & \\
\hline $\operatorname{RBC}\left(x 10^{3} / \mu 1\right)$ & $4.10 \pm 0.50$ & $4.08 \pm 0.51$ & 0.462 \\
\hline $\mathrm{Hb}(\mathrm{g} / \mathrm{dl})^{\mathrm{c}}$ & $10.28 \pm 1.73$ & $12.02 \pm 1.87$ & $0.027^{\mathrm{a}}$ \\
\hline $\operatorname{HCT}(\%)^{\mathrm{c}}$ & $32.30 \pm 4.62$ & $32.47 \pm 4.86$ & 0.892 \\
\hline $\operatorname{MCV}(\mathrm{fl})^{\mathrm{c}}$ & $78.63 \pm 4.14$ & $79.65 \pm 4.57$ & $0.027^{\mathrm{a}}$ \\
\hline $\mathrm{MCH}(\mathrm{pg} / \text { cell })^{\mathrm{c}}$ & $24.95 \pm 1.79$ & $29.37 \pm 1.71$ & $0.028^{\mathrm{a}}$ \\
\hline Platelet $\left(\times 10^{3} / \mu 1\right)^{\mathrm{c}}$ & $240.00 \pm 40.41$ & $250.67 \pm 36.06$ & 0.345 \\
\hline $\mathrm{WBC}\left(\times 10^{3} / \mu 1\right)^{\mathrm{b}}$ & $5.50 \pm 2.51$ & $5.65 \pm 2.32$ & 0.651 \\
\hline Neutrophils $(\%)^{\mathrm{c}}$ & $58.78 \pm 9.63$ & $61.47 \pm 8.90$ & 0.173 \\
\hline Eosinophils+Basophils (\%) & $6.27 \pm 1.51$ & $7.48 \pm 1.80$ & 0.156 \\
\hline $\operatorname{ALT}(\mathrm{U} / \mathrm{l})^{\mathrm{c}}$ & $19.17 \pm 9.87$ & $18.50 \pm 8.14$ & 0.461 \\
\hline $\operatorname{AST}(\mathrm{U} / \mathrm{l})^{\mathrm{c}}$ & $30.00 \pm 8.10$ & $27.83 \pm 6.47$ & $0.027^{\mathrm{a}}$ \\
\hline $\mathrm{UA}(\mathrm{mg} / \mathrm{dl})^{\mathrm{c}}$ & $5.71 \pm 2.70$ & $5.88 \pm 2.65$ & 0.046 \\
\hline
\end{tabular}

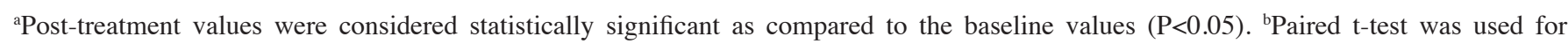
normally distributed data. ${ }^{c}$ Wilcoxon Signed Rank test for related samples was used for non-normally distributed data. RBC, red blood cell; Hb, hemoglobin; HCT, hematocrit; MCV, mean corpuscular volume; $\mathrm{MCH}$, mean corpuscular hemoglobin; WBC, white blood cell; ALT, alanine aminotransferase; AST, aspartate aminotransferase; UA, uric acid.

Table II. Hematological and biochemical characteristics (blood parameters, liver enzymes, and kidney function) of geriatric subjects in the Placebo group.

\begin{tabular}{|c|c|c|c|}
\hline \multirow[b]{2}{*}{ Parameters } & \multicolumn{2}{|c|}{ Placebo Group } & \multirow[b]{2}{*}{ P-value } \\
\hline & Baseline & 1 Month & \\
\hline $\operatorname{RBC}\left(x 10^{3} / \mu 1\right)^{\mathrm{d}}$ & $4.50 \pm 0.47$ & $4.59 \pm 0.45$ & 0.575 \\
\hline $\mathrm{Hb}(\mathrm{g} / \mathrm{dl})^{\mathrm{d}}$ & $11.93 \pm 1.67$ & $13.82 \pm 1.44$ & $<0.001^{\mathrm{a}}$ \\
\hline $\operatorname{HCT}(\%)^{\mathrm{e}}$ & $36.20 \pm 4.53$ & $36.68 \pm 3.52$ & 0.463 \\
\hline MCV (fl) & $80.65 \pm 5.76$ & $80.20 \pm 4.62$ & 0.885 \\
\hline $\mathrm{MCH}(\mathrm{pg} / \mathrm{cell})^{\mathrm{e}}$ & $26.47 \pm 2.15$ & $30.12 \pm 1.84$ & $0.028^{\mathrm{a}}$ \\
\hline Platelet $\left(\times 10^{3} / \mu \mathrm{l}\right)^{\mathrm{d}}$ & $217.17 \pm 50.92$ & $231.50 \pm 48.03$ & 0.185 \\
\hline $\mathrm{WBC}\left(\times 10^{3} / \mu 1\right)^{\mathrm{e}}$ & $5.37 \pm 1.47$ & $6.05 \pm 1.24$ & $0.043^{\mathrm{b}}$ \\
\hline Neutrophils (\%) & $53.43 \pm 17.26$ & $57.57 \pm 11.62$ & 0.321 \\
\hline Eosinophils+Basophils (\%) & $6.25(5.85-8.2)^{c}$ & $7.65(6.68-22.95)^{\mathrm{c}}$ & 0.345 \\
\hline $\operatorname{ALT}(\mathrm{U} / \mathrm{l})^{\mathrm{d}}$ & $23.67 \pm 12.80$ & $20.33 \pm 8.36$ & 0.147 \\
\hline $\operatorname{AST}(\mathrm{U} / 1)^{\mathrm{d}}$ & $20.17 \pm 6.55$ & $18.33 \pm 5.82$ & 0.069 \\
\hline $\mathrm{UA}(\mathrm{mg} / \mathrm{dl})^{\mathrm{d}}$ & $8.24 \pm 3.74$ & $8.17 \pm 3.81$ & 0.508 \\
\hline
\end{tabular}

apost-treatment values were considered statistically significant as compared to the baseline values $(\mathrm{P}<0.05) .{ }^{\mathrm{b}}$ The observed increase in the $\%$ $\mathrm{WBC}$ in the placebo group is within the normal range in adults and aged humans and therefore was considered insignificant (18-44\%). ${ }^{\mathrm{C}} \mathrm{Median}$ and IQR are presented. ${ }^{\mathrm{d}}$ Paired t-test was used for normally distributed data. ${ }^{\mathrm{e} W i l c o x o n ' s ~ S i g n e d ~ R a n k ~ t e s t ~ w a s ~ u s e d ~ f o r ~ n o n-n o r m a l l y ~ d i s t r i b-~}$ uted data. RBC, red blood cell; Hb, hemoglobin; HCT, hematocrit; MCV, mean corpuscular volume; MCH, mean corpuscular hemoglobin; WBC, white blood cell; ALT, alanine aminotransferase; AST, aspartate aminotransferase; UA, uric acid.

Age-related decline in NK activity is associated with decreased lytic activity per cell $(31,32)$ and drops in the expression of granular perforins, which are primarily responsible for destroying cancer and virally infected cells, a characteristic of both NK cells and cytotoxic T lymphocytes (32). The preliminary data in this study shows that daily intake of Biobran/MGN-3 supplement at a dose of $500 \mathrm{mg} /$ day for 1 month increases NK cell activity in the geriatric population. The underlying mechanism is not known but may be attributed to an increase in NK granular perforins and Granzyme-B $(25,27)$, or due to the ability of Biobran/MGN-3 to influence the early signaling events leading to the triggering of protein kinase $\mathrm{C}(30)$. 
Table III. Effect of Biobran/MGN-3 and placebo supplementation on percentages of lymphocyte, NK, and NKT cells within each group before and after treatment.

\begin{tabular}{llccc}
\hline Treatment & Parameters & Baseline & 1 Month & P-value \\
\hline Biobran/MGN-3 & Lymph (\%) & $32.25 \pm 6.05$ & $36.58 \pm 7.77$ & 0.157 \\
& NK (\%) & $4.73 \pm 1.69$ & $7.02 \pm 3.94$ & 0.102 \\
NKT (\%) & $2.72 \pm 0.98$ & $3.6 \pm 2.03$ & 0.256 \\
Placebo & Lymph (\%) & $33.33 \pm 10.19$ & $39.52 \pm 9.82$ & $0.014^{\mathrm{a}}$ \\
& NK (\%) & $4.83 \pm 2.18$ & $10.97 \pm 5.5$ & $0.013^{\mathrm{a}}$ \\
& NKT (\%) & $4.18 \pm 1.66$ & $5.55 \pm 2.44$ & 0.271 \\
\hline
\end{tabular}

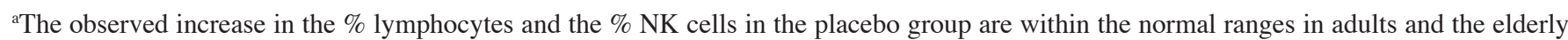
and therefore considered clinically not significant (18-44 and 7-28\%, respectively). The 2-sided paired t-test was used to calculate P-values. NK, natural killer; NKT, natural killer T.

Table IV. Comparison of CD107a expression on activated NK and NKT cells within each group before and after treatment.

\begin{tabular}{|c|c|c|c|c|c|}
\hline Variable & Group & $\begin{array}{l}\text { Pre- } \\
\text { treatment } \\
\text { median }\end{array}$ & $\begin{array}{l}\text { Post- } \\
\text { treatment } \\
\text { median }\end{array}$ & $\begin{array}{c}\text { Wilcoxon } \\
\text { Singed Rank } \\
\text { test Z-value }\end{array}$ & $\begin{array}{c}\text { Asym. Sig. } \\
\text { (2-tailed) } \\
\text { P-value }\end{array}$ \\
\hline \multirow[t]{2}{*}{ PMA/Ionomycin stimulated NK 107a } & Biobran/MGN-3 & 60.5 & 83.0 & -1.992 & $0.046^{\mathrm{a}}$ \\
\hline & Placebo & 40.9 & 54.35 & -0.734 & 0.463 \\
\hline \multirow[t]{2}{*}{ PMA/Ionomycin non-stimulated NKT 107a } & Biobran/MGN-3 & 78.0 & 88.55 & -0.734 & 0.463 \\
\hline & Placebo & 69.0 & 84.0 & -0.943 & 0.345 \\
\hline
\end{tabular}

aPost-treatment values were considered statistically significant as compared to the pre-treatment values $(\mathrm{P}<0.05)$. NK, natural killer; NKT, natural killer T ; Asym. Sig., asymptotic significance.

Aged subjects in both groups (Biobran/MGN-3 and placebo) showed similar insignificant changes in the levels of CD107a expression on non-PMA/Ionomycin-stimulated NK cells at one-month post-treatment relative to baseline values. However, Biobran/MGN-3 supplementation significantly upregulated the percent of PMA/Ionomycin-stimulated NK cells expressing CD107a compared to the baseline value and the placebo group (Fig. 1). Biobran/MGN-3 supplementation upregulation of NK expressing CD107a is also well illustrated in flow cytometry depicted in Fig. 2B. Subjects in the placebo group did not show upregulated PMA/Ionomycin-induced NK expressing CD107a; CD107a was positive only in very few NK cells (Fig. 2A). The increase in activity post-treatment with Biobran/MGN-3 was not associated with the percent of NK cells. Data in Table III showed that there was no significant change in \% lymphocytes and $\%$ NK cells in the Biobran/MGN-3 or placebo group. These data are in accordance with the work of others, who show the normal range for lymphocytes from healthy subjects is between $18-44 \%$ (33) and the normal range for NK cells is between $7-28 \%(34,35)$. The NK cell expression of CD107a has been reported to be parallel to the increase in the cytotoxic effect of NK cells (22).

Recent research showed CD107a is not merely a marker of degranulation but it plays a critical role in lysosome formation and the degranulation process. RNAi-mediated silencing of $\mathrm{CD} 107 \mathrm{a}$ expression in NK cells resulted in failure to deliver

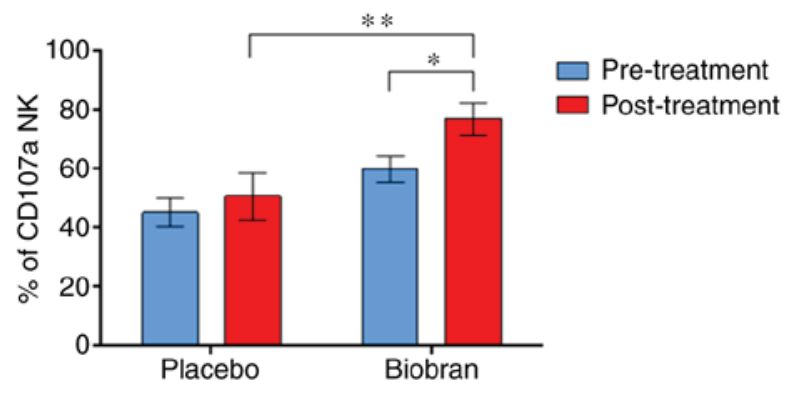

Figure 1. Effect of Biobran/MGN-3 on the percentage of chemically-activated NK cells that express CD107a. Biobran/MGN-3 supplementation for 1 month significantly increased the percentage of CD107a-expressing NK cells that had been chemically activated with PMA/Ionomycin treatment. Bar graph represents medians $\pm \mathrm{IQR}$. Wilcoxon's signed rank and Mann-Whitney tests were used to compare within (before and after) and between (Biobran/MGN-3 vs. placebo) groups, respectively. No significant difference between the pre-treatment levels in the two groups was observed. "Significantly higher than PMA/Ionomycin-stimulated NK cells before treatment supplementation $(\mathrm{P}<0.05){ }^{* * *}$ Significantly higher than PMA/Ionomycin-stimulated NK cells in the treatment of placebo group $(\mathrm{P}<0.05)$. NK, natural killer.

Granzyme B to target cells, impaired perforin trafficking, decreased perforin content in lytic granules, and impaired NK cell cytotoxic activity (36). On the other hand, increased expression of CD107a has been shown to correlate with cytokine secretion and cytotoxic activity of NK cells (37). Thus, our 


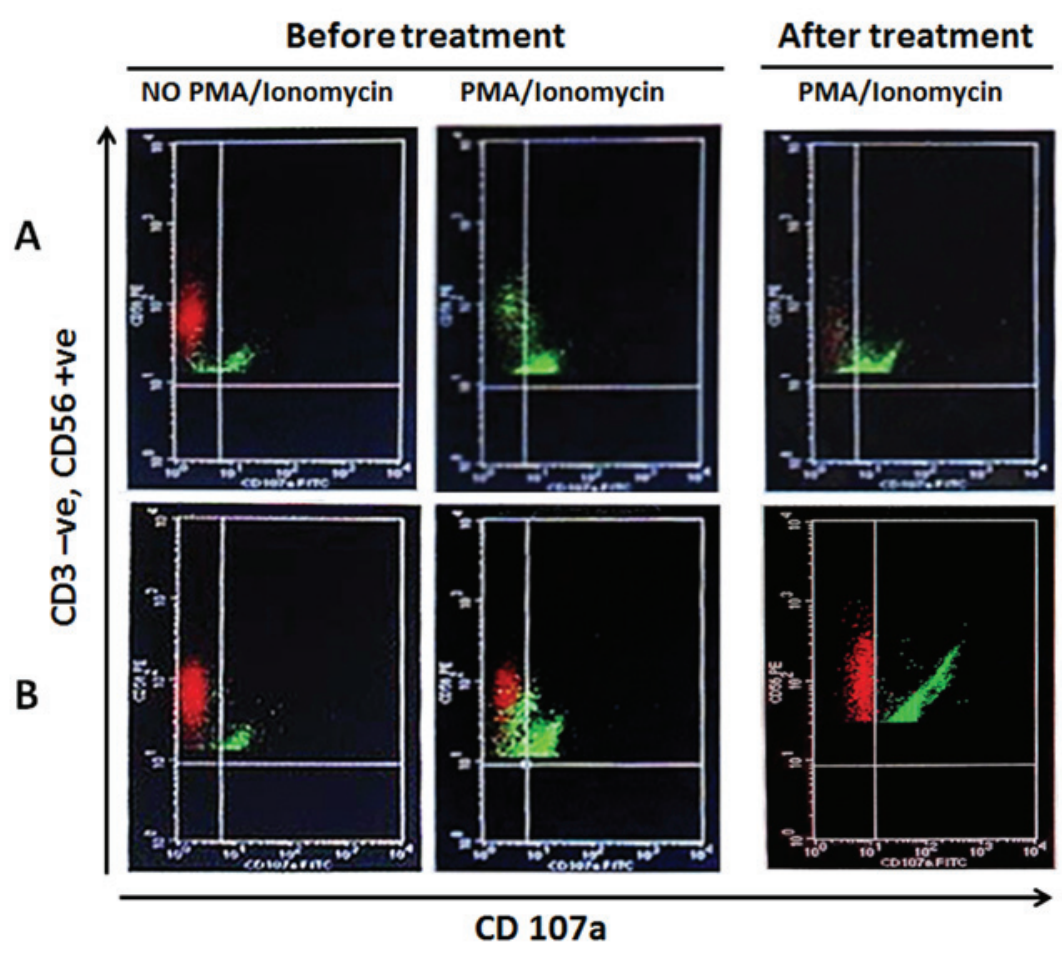

Figure 2. Flow cytometry analysis comparing the effects of (A) placebo vs. (B) Biobran/MGN-3 supplementations on CD107a expression in PMA/Ionomycin-stimulated NK cells and non-PMA/Ionomycin-stimulated NK cells. Biobran/MGN-3 supplementation for 1 month significantly increased the number of CD107a-expressing NK cells upon PMA/Ionomycin stimulation (lower right), compared to both PMA/Ionomycin-stimulated NK cells before Biobran/MGN-3 supplementation (lower middle) and PMA/Ionomycin-stimulated NK cells after 1 month of placebo treatment (upper right). CD107a expression levels in PMA/Ionomycin-stimulated and non-PMA/Ionomycin-stimulated NK cells were not significantly different in either the placebo or Biobran/MGN-3 groups prior to treatment (middle and left, respectively). NK, natural killer.

results suggest that Biobran/MGN-3 supplement increases lytic granules trafficking, degranulation capacity, and cytotoxicity of NK cells. NK cells have been considered to be a bridge between the innate response and the adaptive system (38), and their activation will lead to modulating other immune cell subsets. This conclusion has been demonstrated in our earlier studies, which showed the ability of Biobran/MGN-3 to modulate human CD8 ${ }^{+}$ T cells, CD4 ${ }^{+} \mathrm{T}$ cells, and B cells post-ingestion (14).

Natural killer T (NKT) cells are a subset of regulatory lymphocytes that co-express cell surface receptors characteristic of both $\mathrm{T}$ lymphocytes (e.g., CD3, $\alpha / \beta$ T-cell receptor [TCR]) and NK cells (e.g., CD56, NK1.1) (39). The reason that Biobran/MGN-3 induces PMA/Ionomycin-stimulated NK cells expressing CD107a and does not induce NKT cells in geriatric subjects is not fully understood, but could be attributed to differences in the cell receptors of NK cells and NKT cells. Our earlier study showed that Biobran/MGN-3 supplementation in vitro for 16 h upregulates three key cell surface receptors on NK cells: CD69, an early activation antigen; CD25, an interleukin-2 receptor; and CD54 (ICAM-I), an adhesion molecule (15). NKT cells are a population of $\mathrm{T}$ cells that are activated by lipid antigens bound to CD1d (cluster of differentiation 1d) molecules at the surface of antigen-presenting cells. There are five different CD1 genes (CD1a-e) that have been identified. CD1a-c proteins are recognized by diverse conventional $\alpha \beta$ T cells, while CD1d proteins are recognized by a specialized subset of $\alpha \beta \mathrm{T}$ cells. The intracellular metabolic pathways of lipid antigens are key in forming the functional NKT cell repertoire $(40,41)$. We believe that more analysis of the lipid nature of these cells is needed in order to assess their response to the immunomodulatory effect of Biobran/MGN-3.

Results of this study showed that dietary supplement Biobran/MGN-3 activates NK cell activity in geriatric subjects. A direct relation between diet and changes in the brain structure and activity has been reported in the guidelines of the National Institute on Aging-Alzheimer's Association. Growing experimental evidence suggests that synapses may be the locus for abnormalities underlying diseases such as Alzheimer's, Parkinson's, and multiple sclerosis. Indeed, perturbations in the induction, maintenance, or reversal of long term potentiation (LTP) and long term depression (LTD) are a common thread in

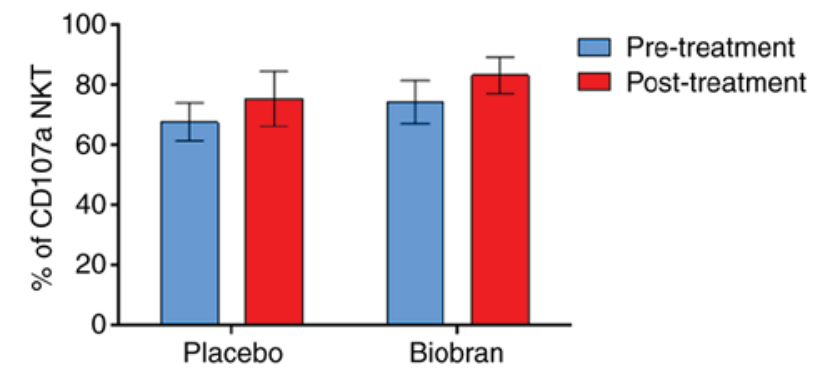

Figure 3. Effect of Biobran/MGN-3 on the percentage of CD107a-expressing NKT (NKT-cells), which were chemically stimulated with PMA/Ionomycin. Biobran/MGN-3 supplementation did not induce significant statistical change in the percentage of CD107a-expressing NKT cells that were chemically activated with PMA/Ionomycin compared to the basal level or to the placebo group. Bar graph represents medians \pm IQR. Wilcoxon's signed rank and Mann-Whitney tests were used to compare within (before and after) and between (Biobran/MGN-3 vs. placebo) groups, respectively. NKT, natural killer T. 
the different brain disease models $(42,43)$, as well as in human pathologies associated with inflammation (44). Thus, it has been suggested that the etiopathogenesis of different conditions is a result of the combination of abnormal expression of immune mediators with other disease-specific features (5). It is of interest to note that the dietary supplement L-Tryptophan prevents the age-induced decline of hippocampal serotonin (5-HT) production and thus avoids cognitive decline (45), and that treatment with sildenafil, a phosphodiesterase-5 inhibitor, resulted in an improvement in hippocampal synaptic dysfunction and memory deficits in Alzheimer's disease mouse model (46). Results of our study and those of others may lead to the design of immunoneurological-targeted therapies.

It is of special interest to note that the NK immunomodulatory effect of Biobran/MGN-3 in geriatric subjects was achieved at a low dose of $500 \mathrm{mg} /$ day. Aged subjects who participated in this randomized, double-blind, clinical trial were healthy, did not complain of any disease, and did not ingest other immune modulators, suggesting the great response of healthy aged subjects to the NK immunomodulatory effect of Biobran/MGN-3. Data in the present study showed Biobran/MGN-3 to be safe and that treatment did not induce any significant toxic changes in any of the studied parameters. Instead, some beneficial effects were observed after 1 month of treatment in the Biobran/MGN-3 group, and the placebo group to a lesser extent. These effects included improved levels of $\mathrm{Hb}, \mathrm{MCH}$, and ALP. In addition, Biobran/MGN-3 supplementation significantly down-regulated AST (SGOT) levels, suggesting improved liver functions. This finding is consistent with our recent study that shows the ability of Biobran/MGN-3 to improve liver functions as indicated by a significant decrease of viremia levels in Hepatitis C patients post-treatment with Biobran/MGN-3 (47). Biobran/MGN-3 has been shown to be a safe and nontoxic agent in several toxicity studies including: i) the subchronic toxicity study in beagle dogs, the guinea pig antigenicity study, and mutagenicity testing $(48,49)$; ii) the LD50 (lethal dose, $50 \%$ ) of Biobran/MGN-3 is greater than $36 \mathrm{~g} / \mathrm{kg}$; and iii) the Ames test for mutagenicity was negative. Furthermore, studies among cancer patients treated with chemotherapy have shown a marked improvement in appetite and other quality of life (QOL) parameters $(50,51)$.

The placebo group in this study ingested dietary fiber, hydroxy propyl distarch phosphate (HDP), which is currently used as a food additive. Data depicted in Tables II and III demonstrate that subjects who ingested HDP showed significantly improved levels of $\mathrm{Hb}$ and $\mathrm{MCH}$ relative to their baseline values. Earlier studies have showed the beneficial role of dietary fiber in overall health. For example, subjects who ingested an HDP meal showed significantly lower postprandial glucose, insulin, and GIP responses than subjects who ingested a waxy maize starch meal (52). Additionally, different types of starch have been shown to influence the retention of 59Fe (53) and absorption of zinc and iron (54). One limitation of our study was the small number of our sample. Studies with larger sample sizes are needed to validate our results. Another limitation was our limited ability to study the full range of molecular markers that are needed to identify potential mechanisms involved in Biobran/MGN-3-induced activation of NK but not NKT cells.

In conclusion, the preliminary results of this study strongly suggest that Biobran/MGN-3, a nutritional supplement from rice bran, can counteract NK cell immunosenescence in geriatric subjects, suggesting the potential of Biobran/MGN-3 for lowering the incidence of cancer and viral infection that is reported by older humans. To our knowledge, the current study represents the first clinical trial that extends the biological response modifier (BRM) effect of Biobran/MGN-3 to include enhancing NK cell degranulation and cytotoxic activity in geriatric population. Biobran/MGN-3 is a non-toxic, inexpensive supplement, and thus may provide a means to reduce the significant healthcare costs incurred by both healthy and unhealthy geriatric subjects. Our future research directions include conducting multicenter prospective clinical trials to assess the preventive capacity of Biobran/MGN-3 supplementation in the geriatric population to reduce the incidence of infections and tumorigenesis.

\section{Acknowledgements}

The authors would like to acknowledge Daiwa Pharmaceutical Co., Ltd., Tokyo, Japan, for supplying Biobran/MGN-3 for this study. This study was supported in part by NIH-NIMHD grant nos. U54MD007598 and NIH/NCATS, and grant nos. UL1TR000124 and S21 MD000103. The authors would also like to thank Dr. B. J. Winjum at UCLA for assistance in writing the manuscript and Dr. S. Gollapudi at UCI for discussions relevant to experimental design and analysis.

\section{References}

1. Garza A: The Aging Population: The Increasing Effects on Health Care. http://www.pharmacytimes.com/publications/issue/2016/january2016/the-aging-population-the-increasi ng-effects-on-health-care. Accessed January 19, 2016.

2. Alzheimer's Association: 2017 Alzheimer's Disease Facts and Figures. http://www.alz.org/facts/. Accessed August 18, 2017.

3. American Cancer Society Cancer Action Network: The Costs of Cancer. Addressing Patient Costs. https://www.acscan.org/sites/ default/files/Costs\%20of\%20Cancer\%20-\%20Final\%20Web. pdf. Accessed August 18, 2017.

4. Boulanger LM: Immune proteins in brain development and synaptic plasticity. Neuron 64: 93-109, 2009.

5. Nisticò R, Salter E, Nicolas C, Feligioni M, Mango D Bortolotto ZA, Gressens P, Collingridge GL and Peineau S: Synaptoimmunology-roles in health and disease. Mol Brain 10: 26, 2017.

6. Gomez CR, Nomellini V, Faunce DE and Kovacs EJ: Innate immunity and aging. Exp Gerontol 43: 718-728, 2008.

7. Zanussi S, Serraino D, Dolcetti R, Berretta M and De Paoli P: Cancer, aging and immune reconstitution. Anticancer Agents Med Chem 13: 1310-1324, 2013.

8. Pera A, Campos C, López N, Hassouneh F, Alonso C, Tarazona R and Solana R: Immunosenescence: Implications for response to infection and vaccination in older people. Maturitas 82: 50-55, 2015.

9. Ma Y and Fang M: Immunosenescence and age-related viral diseases. Sci China Life Sci 56: 399-405, 2013.

10. Lotzová E, Savary CA, Freedman RS and Bowen JM: Natural killer cell cytotoxic potential of patients with ovarian carcinoma and its modulation with virus-modified tumor cell extract. Cancer Immunol Immunother 17: 124-129, 1984.

11. Moretta L, Bottino C, Pende D, Mingari MC, Biassoni R and Moretta A: Human natural killer cells: Their origin, receptors and function. Eur J Immunol 32: 1205-1211, 2002.

12. Chretien AS, Le Roy A, Vey N, Prebet T, Blaise D, Fauriat C and Olive D: Cancer-induced alterations of NK-mediated target recognition: Current and investigational pharmacological strategies aiming at restoring NK-mediated anti-tumor activity. Front Immunol 5: 122, 2014.

13. Ivarsson MA, Michaëlsson J and Fauriat C: Activating killer cell Ig-like receptors in health and disease. Front Immunol 5: 184, 2014. 
14. Ghoneum M: Anti-HIV activity in vitro of MGN-3, an activated arabinoxylane from rice bran. Biochem Biophys Res Commun 243: 25-29, 1998

15. Ghoneum M and Jewett A: Production of tumor necrosis factor-alpha and interferon-gamma from human peripheral blood lymphocytes by MGN-3, a modified arabinoxylan from rice bran, and its synergy with interleukin-2 in vitro. Cancer Detect Prev 24: 314-324, 2000.

16. Ghoneum $\mathrm{M}$ and Gollapudi S: Modified arabinoxylan rice bran (MGN-3/Biobran) sensitizes human T cell leukemia cells to death receptor (CD95)-induced apoptosis. Cancer Lett 201: 41-49, 2003.

17. Badr El-Din NK, Noaman E and Ghoneum M: In vivo tumor inhibitory effects of nutritional rice bran supplement MGN-3/Biobran on Ehrlich carcinoma-bearing mice. Nutr Cancer 60: 235-244, 2008.

18. Ghoneum M and Agrawal S: Activation of human monocyte-derived dendritic cells in vitro by the biological response modifier arabinoxylan rice bran (MGN-3/Biobran). Int J Immunopathol Pharmacol 24: 941-948, 2011.

19. Cholujova D, Jakubikova J, Czako B, Martisova M, Hunakova L, Duraj J, Mistrik M and Sedlak J: MGN-3 arabinoxylan rice bran modulates innate immunity in multiple myeloma patients. Cancer Immunol Immunother 62: 437-445, 2013

20. Ghoneum M, Badr El-Din NK, Ali DA and El-Dein MA: Modified arabinoxylan from rice bran, MGN-3/biobran, sensitizes metastatic breast cancer cells to paclitaxel in vitro. Anticancer Res 34: 81-87, 2014.

21. Ghoneum M: Enhancement of human natural killer cell activity by modified arabinoxylane from rice bran (MGN-3). Int J Immunothe 14: 89-99, 1998.

22. Alter G, Malenfant JM and Altfeld M: CD107a as a functional marker for the identification of natural killer cell activity. J Immunol Methods 294: 15-22, 2004

23. Ooi SL, McMullen D, Golombick T, Nut D and Pak SC: Evidence-based review of BioBran/MGN-3 arabinoxylan compound as a complementary therapy for conventional cancer treatment. Integr Cancer Ther: Oct 1, 2017 (Epub ahead of print). doi: $10.1177 / 1534735417735379$.

24. Ghoneum M and Brown J: NK immunorestoration of cancer patients by MGN-3, a modified arabinoxylan rice bran (study of 32 patients followed for up to 4 years). In: Wheat and rice in disease prevention and health. Anti-aging medical therapeutics. Klatz R and Goldman R (eds). Vol III. Health Quest Publications, Marina del Rey, CA, pp217-226, 1999.

25. Ghoneum $\mathrm{M}$ and Abedi S: Enhancement of natural killer cell activity of aged mice by modified arabinoxylan rice bran (MGN-3/Biobran). J Pharm Pharmacol 56: 1581-1588, 2004.

26. Pérez-Martínez A, Valentín J, Fernández L, Hernández-Jiménez E, López-Collazo E, Zerbes P, Schwörer E, Nuñéz F, Martín IG, Sallis H, et al: Arabinoxylan rice bran (MGN-3/Biobran) enhances natural killer cell-mediated cytotoxicity against neuroblastoma in vitro and in vivo. Cytotherapy 17: 601-612, 2015

27. Ghoneum M: From bench to bedside: The growing use of arabinoxylan rice bran (MGN-3/Biobran) in cancer immunotherapy. Austin Immunol 1: 1006, 2016.

28. Ogata K, An E, Shioi Y, Nakamura K, Luo S, Yokose N, Minami S and Dan K: Association between natural killer cell activity and infection in immunologically normal elderly people. Clin Exp Immunol 124: 392-397, 2001.

29. Smetana K Jr, Lacina L, Szabo P, Dvořánková B, Brož P and Šedo A Ageing as an important risk factor for cancer. Anticancer Res 36: 5009-5017, 2016.

30. Ghoneum M, Suzuki K and Gollapudi S: Phorbol myristate acetate corrects impaired NK function of old mice. Scand J Immunol 34 391-397, 1991

31. Mariani E, Sgobbi S, Meneghetti A, Tadolini M, Tarozzi A, Sinoppi M, Cattini L and Facchini A: Perforins in human cytolytic cells: The effect of age. Mech Ageing Dev 92: 195-209, 1996.

32. Rukavina D, Laskarin G, Rubesa G, Strbo N, Bedenicki I, Manestar D, Glavas M, Christmas SE and Podack ER: Age-related decline of perforin expression in human cytotoxic T lymphocytes and natural killer cells. Blood 92: 2410-2420, 1998.

33. Chng WJ, Tan GB and Kuperan P: Establishment of adult peripheral blood lymphocyte subset reference range for an asian population by single-platform flow cytometry: Influence of age, sex, and race and comparison with other published studies. Clin Diagn Lab Immunol 11: 168-173, 2004.

34. Wang WS, Lo AW, Siu LP, Leung JN, Tu SP, Tai SW, Lam SC and Wong KF: Reference ranges for lymphocyte subsets among healthy Hong Kong Chinese adults by single-platform flow cytometry. Clin Vaccine Immunol 20: 602-606, 2013
35. Al-Mawali A,Pinto AD,BusaidiRA and Al-ZakwaniI:Lymphocyte subsets: Reference ranges in an age- and gender-balanced population of Omani healthy adults. Cytometry A 83: 739-744, 2013.

36. Krzewski K, Gil-Krzewska A, Nguyen V, Peruzzi G and Coligan JE: LAMP1/CD107a is required for efficient perforin delivery to lytic granules and NK-cell cytotoxicity. Blood 121: 4672-4683, 2013.

37. Aktas E, Kucuksezer UC, Bilgic S, Erten G and Deniz G: Relationship between CD107a expression and cytotoxic activity Cell Immunol 254: 149-154, 2009.

38. Sun JC and Lanier LL: Natural killer cells remember: An evolutionary bridge between innate and adaptive immunity? Eur J Immunol 39: 2059-2064, 2009.

39. Godfrey DI, Hammond KJ, Poulton LD, Smyth MJ and Baxter AG: NKT cells: Facts, functions and fallacies. Immunol Today 21: 573-583, 2000.

40. Brigl M and Brenner MB: CD1: Antigen presentation and T cell function. Annu Rev Immunol 22: 817-890, 2004.

41. Shin JH and Park SH: The effect of intracellular trafficking of CD1d on the formation of TCR repertoire of NKT cells. BMB Rep 47: 241-248, 2014.

42. Nisticò R, Pignatelli M,Piccinin S, Mercuri NB and Collingridge G: Targeting synaptic dysfunction in Alzheimer's disease therapy. Mol Neurobiol 46: 572-587, 2012

43. Pignatelli M, Feligioni M, Piccinin S, Molinaro G, Nicoletti F and Nisticò R: Synaptic plasticity as a therapeutic target in the treatment of autism-related single-gene disorders. Curr Pharm Des 19: 6480-6490, 2013

44. Nisticò R, Mori F, Feligioni M, Nicoletti F and Centonze D: Synaptic plasticity in multiple sclerosis and in experimental autoimmune encephalomyelitis. Philos Trans R Soc Lond Ser B Biol Sci 369: 20130162, 2013.

45. Musumeci G, Castrogiovanni P, Szychlinska MA, Imbesi R, Loreto C, Castorina S and Giunta S: Protective effects of high Tryptophan diet on aging-induced passive avoidance impairment and hippocampal apoptosis. Brain Res Bull 128: 76-82, 2017.

46. Puzzo D, Staniszewski A, Deng SX, Privitera L, Leznik E, Liu S, Zhang H, Feng Y, Palmeri A, Landry DW and Arancio O: Phosphodiesterase 5 inhibition improves synaptic function, memory, and amyloid-beta load in an Alzheimer's disease mouse model. J Neurosci 29: 8075-8086, 2009.

47. Salama H, Medhat E, Shaheen M, Zekri AN, Darwish T and Ghoneum M: Arabinoxylan rice bran (Biobran) suppresses the viremia level in patients with chronic HCV infection: A randomized trial. Int J Immunopathol Pharmacol 29: 647-653, 2016.

48. Daiwa Pharmaceutical and Co., Ltd.: BioBran rice bran arabinoxylan compound. http://www.daiwa-pharm.com/english/product/ biobran.html. Accessed October 22, 2017

49. Tazawa K: BioBran/MGN-3: Basic and Clinical Application to Integrative Medicine. Iyakushuppan Co. Publishers, Tokyo, pp18-22, 2003.

50. Takahara K and Sano K: The life prolongation and QOL improvement effect of rice bran arabinoxylan derivative (MGN-3, Bio-Bran) for progressive cancer. Clin Pharmacol Therapy 14: 267-271, 2004.

51. Hajto T, Horvath A and Papp S: Improvement of quality of life in tumor patients after an immunomodulatory treatment with standardized mistletoe lectin and arabinoxylan plant extracts. Int J Neurorehabilitation 3: 1-3, 2016.

52. Shimotoyodome A, Suzuki J, Kameo Y and Hase T: Dietary supplementation with hydroxypropyl-distarch phosphate from waxy maize starch increases resting energy expenditure by lowering the postprandial glucose-dependent insulinotropic polypeptide response in human subjects. Br J Nutr 106: 96-104, 2011.

53. Hood LF, Vancampen DR, House WA and Szatkowski E: Effect of modified and unmodified tapioca starches on 59Fe retention in rats. J Nutr 106: 1768-1772, 1976

54. Kishida T, Nakai Y and Ebihara K: Hydroxypropyl-distarch phosphate from Tapioca starch reduces zinc and iron absorption, but not calcium and magnesium absorption, in rats. J Nutr 131: 294-300, 2001

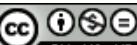

This work is licensed under a Creative Commons Attribution-NonCommercial-NoDerivatives 4.0 International (CC BY-NC-ND 4.0) License. 